\title{
Model gradient coil employing active acoustic control for MRI
}

\author{
B. Haywood - B. Chapman · P. Mansfield
}

Received: 22 January 2007 / Revised: 4 September 2007 / Accepted: 8 October 2007 / Published online: 15 November 2007 (C) ESMRMB 2007

\begin{abstract}
Results are presented for a model three-axis gradient coil incorporating active acoustic control which is applied to the switched read gradient during a single-shot rapid echo-planar imaging (EPI) sequence at a field strength of 3.0 T. The total imaging acquisition time was $10.6 \mathrm{~ms}$. Substantial noise reduction is achieved both within the magnet bore and outside the magnet. Typical internal noise reduction over the specimen area is $40 \mathrm{~dB}_{\mathrm{A}}$ whereas outside the acoustic chamber the noise level is reduced by $60-67 \mathrm{~dB}_{\mathrm{A}}$. However these results are relative to a control winding which is switched in phase, adding $6 \mathrm{~dB}_{\mathrm{A}}$ in its non-optimized mode, which is included in the quoted figures.
\end{abstract}

Keywords MRI - Acoustic control · Gradient coils · Reduced acoustic noise · EPI

\section{Introduction}

With the advent of more-powerful magnets and faster imaging regimes in magnetic resonance imaging (MRI) a major concern is the high levels of noise generated during scanning. This noise arises within the cylindrical structure of the gradient coil and is proportional to the driving current and the strength of the static magnetic field. This current generates Lorentz forces that induce vibrations in the coil structure, thereby generating substantial noise.

The general trend for increased static field strengths and faster imaging techniques have made the noise situation

B. Haywood $(\varangle) \cdot$ B. Chapman · P. Mansfield

Sir Peter Mansfield Magnetic Resonance Centre,

University of Nottingham, University Park,

Nottingham NG7 2RD, UK

e-mail: brett@bhaywood.freeserve.co.uk

B. Chapman

General Magnetic, Strelley Hall, Nottingham, UK worse. The acoustic noise problem has now reached the point where it poses a significant health risk not only to the patient but also to medical staff working in close proximity to scanners. For example, in ultra-high-speed imaging such as echoplanar imaging (EPI) and echo-volumar imaging (EVI), because of the high maximum gradient amplitudes employed and extremely rapid slew rates used in these techniques, noise levels can approach $130 \mathrm{~dB}_{\mathrm{A}}$ [1-3], which is well above the safety levels permitted in the workplace. While adult patients can be protected to some extent against the dangers of acoustic noise, infants and foetuses in utero cannot be so easily protected. The same is true for animals in veterinary practice.

Various approaches have been explored to try to ameliorate the acoustic noise problem, including the development of special pulses for use in EPI [4,5], and also gradient shielding techniques [6]. Presently the most common approach is to seal the gradient coil in a vacuum chamber [7].

As an alternative approach, active acoustic control for gradient coils was introduced by Mansfield and Haywood [8] in an attempt to ameliorate the acoustic noise problem by controlling the vibrational modes generated in the coil structure. In this novel approach the gradient coil structure comprises flat rectangular plates or flat arcuate sectors, in which the gradient field winding and an additional control winding are embedded in the flat plate structure in order to control the vibrational mode of the structure, thereby reducing the noise. The use of either rectangular or arcuate sectors simplifies somewhat the theory of noise propagation.

The purpose of the control winding is to prevent the production of compressional and bending vibration modes in the coil structure that result from the opposing forces applied to the coil former segments by the opposite currents in the gradient and return arcs of the coil. It achieves this by decoupling these two currents. Each coil segment consists of two plates separated by a small gap. Around this gap a second, 
control winding is placed which carries a current $I_{1}^{\prime} \mathrm{e}_{1}^{\mathrm{i} \phi}$ for the plates with a gradient field winding current $I_{1}$, and $I_{2}^{\prime} \mathrm{e}_{2}^{\mathrm{i} \phi}$ for the plates with a gradient field winding current $I_{2}$. The two halves of the former can then move independently, thereby reducing considerably the compressive and bending forces. These forces cannot be eliminated entirely as the former is not infinitely rigid. To assist this process further the control winding is activated so that the currents flowing on each side of both sections are acting in unison. Simple theory indicates that the current and phase in the control windings should be equal to those in the gradient windings. Due to the finite velocity of sound in the formers greater acoustic attenuation is achieved by modifying slightly the amplitude and phase of the current in the control windings.

In the work presented here, as a first step to demonstrating the principle of active acoustic control, a scaled-down model three-axis gradient coil was constructed and used to obtain a single-shot image using EPI. The coil consists of a number of sectors, each comprising flat rectangular plates with embedded straight wire windings for the transverse gradient, $G_{y}$, used as the read gradient, together with embedded straight wire secondary control windings for active acoustic control of this switched gradient. The same method of flat rectangular plates with embedded straight wire gradient windings was used for the $G_{x}$ gradient but without the control winding. The longitudinal gradient, $G_{z}$, used a traditional Maxwell pair.

\section{Magnetic field gradient calculations}

The field gradient in the $z$-direction, $\mathbf{G}$, has components [2]

$$
\begin{aligned}
G_{x} & =\frac{\partial B_{z}}{\partial x}, \\
G_{y} & =\frac{\partial B_{z}}{\partial y}, \\
G_{z} & =\frac{\partial B_{z}}{\partial z} .
\end{aligned}
$$

In our coordinate system $G_{x}$ is the equivalent to the generation of $G_{y}$ but with rotation of the gradient assembly through $90^{\circ}$ about the $z$-axis. In this work we concern ourselves entirely with the generation of $G_{y}$.

A magnetic field gradient, $G_{y}$, of the required strength and extent can be produced by at least four parallel straight wires placed at the corners of a rectangle with all wires carrying equal currents flowing in the same direction. By increasing the number of parallel wire pairs and by appropriately changing the spacing of the wires along the $z$-axis the region of linearity of the field gradient produced can be enlarged.

Starting with the impractical infinite wire case for our calculations, following Zupancic and Pirš [9], we express the $z$-component of the magnetic field for six wires, each carrying current $I$, as

$B_{z}(y, z)=\frac{\mu_{0} I}{4 \pi} \operatorname{Re} \sum_{n=0}^{\infty}\left(\frac{\xi}{r}\right)^{n} A_{n}$,

where

$A_{n}=\exp [-\mathrm{i}(n+1) \phi]$,

where the displacement $\xi$ in Eq. (2) is given by

$\xi=y+\mathrm{i} z$,

and where $r$ and $\phi$ are the cylindrical coordinates of a wire, and $y$ and $z$ are the coordinates of a point at which the field is calculated.

If Eq. (2) is expanded one can readily see that all even powers of $\xi$ vanish. When $z=0$ the first term in Eq. (2), when differentiated with respect to $y$, yields a linear gradient $G_{y}$. For six wires, the region of linearity can be made larger when both $A_{3}$ and $A_{5}$ are zero.

Let the current in the corner wires be $I_{1}$, and that in the center wires be $I_{2}$. We find, after some calculation, that $I_{2}: I_{1}$ is $0.8: 1$ with an angle $\delta$ between the diagonals of the corner plate pairs of $66.6^{\circ}$.

An infinite wire design is impractical. All wires that form the windings have to fit inside the magnet, as do the control wires.

Field of finite parallel wires

The optimum angle between the diagonal corner wires for an infinite wire system has been calculated. We now use this angle in magnetic field gradient calculations for a practical coil design with finite wire lengths. The magnetic field $\mathbf{B}(\mathbf{r})$ at a distance $\mathbf{r}$ from a wire displaced $\mathbf{r}_{\mathrm{w}}$ from the origin and which carries a current $I$ along a wire path $w$ is given by the Biot-Savart expression

$\mathbf{B}(\mathbf{r})=\frac{\mu_{0} I}{4 \pi} \int_{\mathrm{w}} \frac{\left(\mathbf{r}-\mathbf{r}_{\mathbf{w}}\right) \times \mathrm{d} \mathbf{r}_{\mathbf{w}}}{\left|\mathbf{r}-\mathbf{r}_{\mathbf{w}}\right|^{3}}$,

where $\mu_{0}$ is the free-space magnetic permeability and $\mathrm{d} \mathbf{r}_{\mathrm{w}}$ is a wire element.

The vector displacements required later are written in Cartesian coordinates as

$\mathbf{r}=\mathbf{i} x=\mathbf{j} y=\mathbf{k} z$

and

$\mathbf{r}_{\mathbf{w}}=\mathbf{i} x_{w}+\mathbf{j} y_{w}+\mathbf{k} z_{w}$.

For a finite wire parallel to the $x$-axis the elements $\mathrm{d} y_{\mathrm{w}}=0$ and $\mathrm{d} z_{\mathrm{w}}=0$. The $B_{x}$ component of Eq. (5) is

$B_{x}(x, y, z)=0$. 


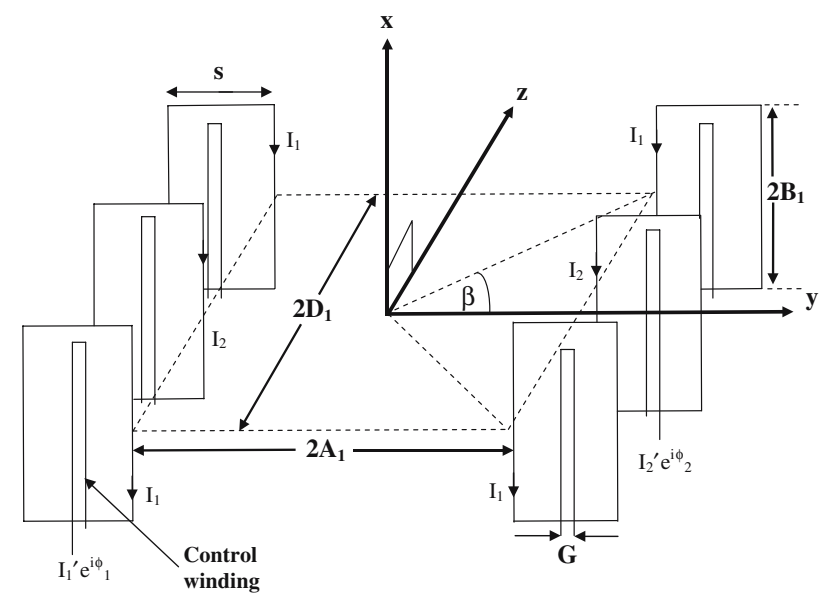

Fig. 1 Sketch showing the layout for the six-sector $G_{y}$ coil assembly. The dimensions $2 D_{1}, 2 A_{1}$, and $2 B_{1}$ are defined in the text. The currents $I_{1}$ and $I_{2}$, corresponding to the outer four plates and the two inner plates, are defined in the text. The control currents $I_{1}^{\prime}$ are associated with the four outer plates and $I_{2}^{\prime}$ with the two inner plates. The gap width $G$ between the control windings is $5 \mathrm{~mm}$

Similarly the $B_{y}$ and $B_{z}$ components are

$B_{y}(x, y, z)=\frac{\mu_{0} I}{4 \pi}\left(z-D_{1}\right) g$

and

$B_{z}(x, y, z)=\frac{\mu_{0} I}{4 \pi}\left(y-A_{1}\right) g$,

where the current $I=1,000 \mathrm{~A}$, the permeability of free space $\mu_{0}=4 \pi \times 10^{-7} \mathrm{NA}^{-2}, D_{1}=A_{1} \tan \beta$ is the spacing along the $z$-axis between the wires in the plate pairs, and $A_{1}$ is the distance between the wires in the $y$-axis to the center (see Fig. 1).

The integral $g$ is given by

$g=\int_{-B_{1}}^{B_{1}} \frac{\mathrm{d} x_{\mathrm{W}}}{\left[\left(x-x_{\mathrm{W}}\right)^{2}+\left(y-A_{1}\right)^{2}+\left(z-D_{1}\right)^{2}\right]^{3 / 2}}$,

and can be solved exactly [9], giving

$$
\begin{aligned}
\mathrm{g}= & \frac{\left(\mathrm{x}+B_{1}\right) /\left[\left(x+B_{1}\right)^{2}+\left(y-A_{1}\right)^{2}+\left(z-D_{1}\right)^{2}\right]^{1 / 2}}{\left(y-A_{1}\right)^{2}+\left(z-D_{1}\right)^{2}} \\
& -\frac{\left(x-B_{1}\right) /\left[\left(x-B_{1}\right)^{2}+\left(y-A_{1}\right)^{2}+\left(z-D_{1}\right)^{2}\right]^{1 / 2}}{\left(y-A_{1}\right)^{2}+\left(z-D_{1}\right)^{2}} .
\end{aligned}
$$

By combining Eqs. (9) and (10) with Eq. (12) and with appropriate values of $A_{1}$ and $D_{1}$, we obtain the magnetic field of six parallel finite length wires. The component $B_{x}$ is zero everywhere and the component $B_{y}$ is zero over the $x y$-plane, which can be chosen as the imaging plane.

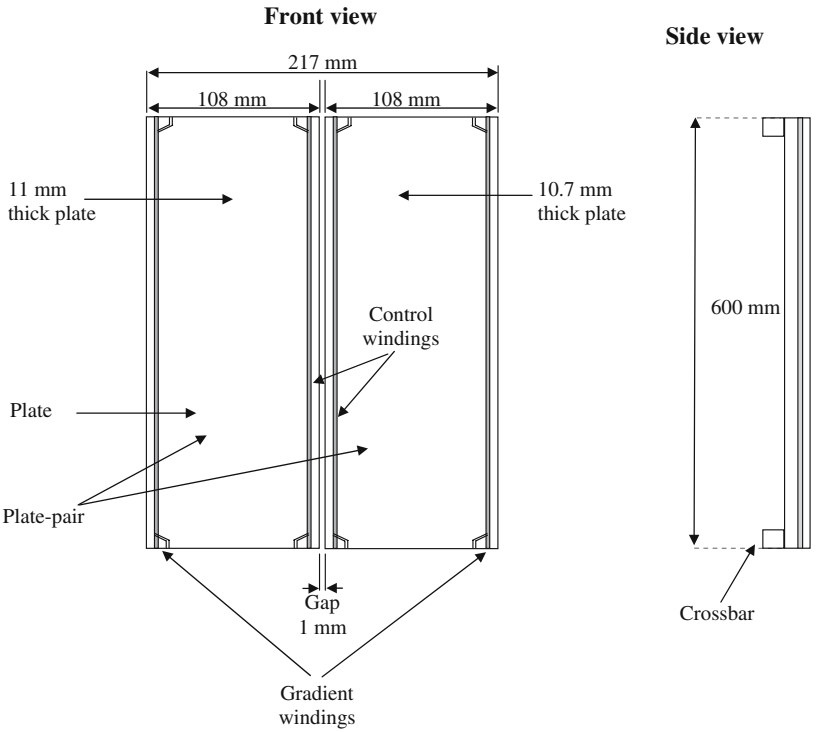

Fig. 2 Diagram of the front and side elevation of the gradient coil plate pair, clearly showing the wire paths for the main field gradient windings and control windings with a $1 \mathrm{~mm}$ air gap between the plates

Gradient field strength and linearity

The gradient strength and linearity were calculated for the $G_{y}$ gradient coil design, comprising six current-carrying loops together with six control winding loops. The coil thickness was given by $2 D_{1}=2 A_{1} \tan \beta$. The length of the plates $2 B_{1}$ was fixed at $\pm 0.30 \mathrm{~m}$. The width of the plate pairs, $s$, was fixed at $0.217 \mathrm{~m}$, this value being the maximum allowable in the magnet. The optimum angle, $\beta$, for the wire positions was also fixed to give maximum linearity. Figure 1 shows a sketch of the $G_{y}$ gradient coil arrangement and a detailed sketch of an individual plate pair or sector making up the coil is shown in Fig. 2. A plot of the calculated magnetic field strength and gradient linearity is shown in Fig. 3.

\section{Effect of the control windings}

Calculations were carried out to determine whether the additional gradient field produced by the control windings would compromise the magnetic efficacy of the $G_{y}$ gradient. The calculations showed that the strength of the field produced by the control windings is determined by the size of the gap between them. By keeping the gap as small as possible, the field produced by the control winding is kept small compared to the $G_{y}$ gradient field. From calculations for our coil configuration, with a gap of $0.005 \mathrm{~m}$ between the control windings, it was found that the control windings added only $3.5 \%$ to the $G_{y}$ gradient field.

The control winding current and relative phase are equal to those supplied to the $G_{y}$ gradient when the coil is operating in the noise cancellation mode. From the results of the 


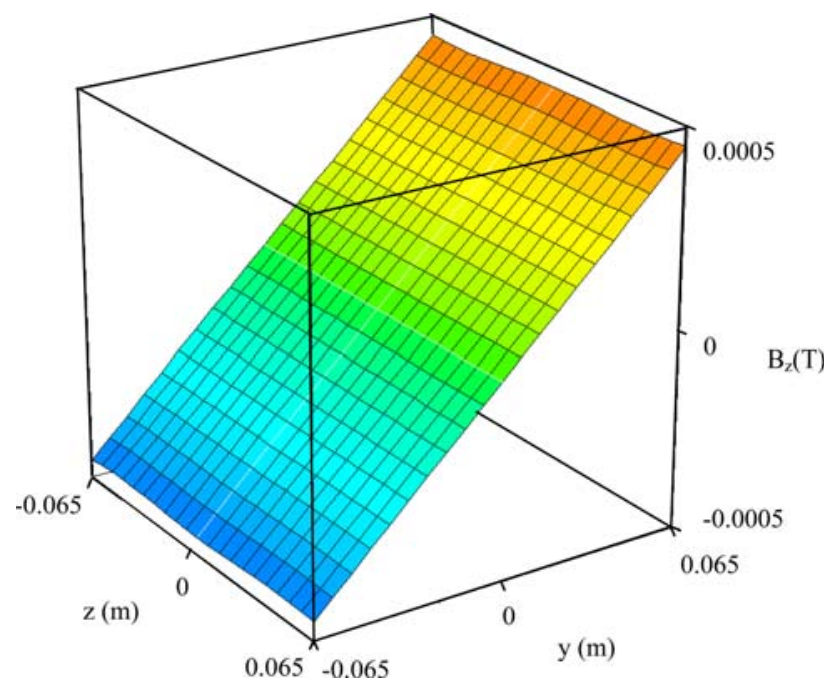

Fig. 3 Computer-generated plot showing the gradient performance over a central region of $6.5 \times 6.5 \mathrm{~cm}^{2}$

calculations above, the small contribution from the control winding to the overall gradient field affects the linearity of the $G_{y}$ gradient field by a negligible amount and stays within $1.5 \%$ over the region of interest (ROI) (see Fig. 3).

Field of view and resolution

To match the acoustically controlled gradient coil system with the imaging parameters of the $3 \mathrm{~T}$ system a field of view (FOV) of $6.4 \mathrm{~cm}^{2}$ with a resolution of $2 \mathrm{~mm}$ along both image axes was chosen.

Fabrication of the gradient coil plate pairs

The flat plates used for the actively acoustically controlled transverse read gradient, $G_{y}$, were constructed by cutting sheets of approximately 11.5 -mm-thick Tufnol $10 \mathrm{G} 40$ glass fabric-epoxy resin laminated plastic to the required dimensions. The plate resonant frequency varied linearly with the plate thickness and served as a way to tune all the plates to a common resonant frequency. Figure 4 shows a frequency sweep and acoustic response of a plate pair of equal thickness. To broaden the resonant peak and give a larger operating area half the plates were machined down to a thickness of $11 \mathrm{~mm}$ and the other half were machined down to a thickness of $10.7 \mathrm{~mm}$ so that, when placed together to form a plate pair, they would have the desired frequency response. Grooves $2.5 \mathrm{~mm}$ wide and $6.0 \mathrm{~mm}$ deep were machined into each half of the plate pair along both edges so that the gradient winding could be inserted along the outer edge and the control winding on the inner edge of the front face of the plate pair. Two layers of round $2 \mathrm{~mm}$ diameter shellacked copper wire were placed on top of each other into each groove and then

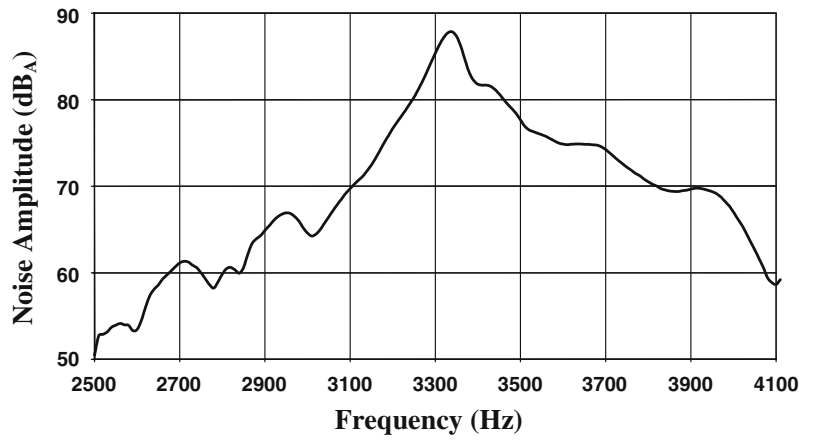

Fig. 4 The acoustic output for a plate pair over a restricted range of $2.5-4.1 \mathrm{kHz}$. Note the peak at around $3.31 \mathrm{kHz}$

covered with approximately $2 \mathrm{~mm}$ of epoxy resin to embed the wires into the plates. The plate pairs were then assembled by placing two plates, one $11 \mathrm{~mm}$ and one $10.7 \mathrm{~mm}$ thick, together. To hold individual plate halves together as plate pairs, 30-mm-wide cross-bars were fitted at the back of the plate pairs with a $1.0 \mathrm{~mm}$ gap between the plates, running the length of the plates. The wire ends on the plates were then soldered together to form a single continuous two-turn outer winding to provide the main gradient field and a continuous two-turn inner winding to provide acoustic control. A diagram of the plate-pair arrangement is shown in Fig. 2. The response of each plate pair was measured acoustically in the magnet. The results of each plate pair showed a doublepeaked response in which the amplitudes of each plate pair differed slightly. However there were areas in each plate pair response where the amplitudes overlapped and would be ideally suited for a common operating frequency. For all six plate pairs the overlap occurred at around $3.2 \mathrm{kHz}$, so an operating frequency of $3.205 \mathrm{kHz}$ was chosen, as it corresponded to one of the MR system's digital sampling frequencies.

\section{Experimental procedure}

With the three banks of gradient amplifiers available on the 3.0 T system, demonstration of the imaging capabilities of the coil at the same time as application of acoustic control was not possible. The high gradient field strength required for such short imaging times meant that large currents were required for imaging. These could only be achieved by employing a bank of eight Techrons to power the gradients via a resonant circuit. This circuit consisted of a bank of high-power capacitors connected in series with the gradient coil windings. At our operating frequency of $3,205 \mathrm{~Hz}$ and with the measured inductance of $52 \mu \mathrm{H}$ ideally we required a capacitance of $47.4 \mu \mathrm{F}$ to tune the coil. With the capacitors available we were able to achieve a measured capacitance of $45.4 \mu \mathrm{F}$, which was sufficient to achieve the required current in the switched gradient coil. 
With only three sets of power supplies and one bank of capacitors available it was almost impossible to carry out noise reduction experiments while imaging, without dispensing with slice selection thus freeing the third bank of Techrons to supply power to the acoustic control winding. As active acoustic control requires almost identical currents in both the control and gradient wires it was necessary to reduce the imaging field to approximately one third of its original size in order to compensate for the lower power capability in the control winding amplifier that consisted of only four Techrons with no second bank of capacitors to operate them in resonant mode. This would have meant most of the image detail would be lost. Consequently the experiment had to be split into two parts. The first part was to obtain an image from the coil utilizing all three gradient axes, but with no acoustic noise cancellation. The second part, after reconfiguring the gradient amplifiers, was the noise cancellation experiment.

The whole three-axis gradient coil assembly, including the cylindrical frame, the six-plate $G_{y}$ gradient coil arrangement, the four-plate $G_{x}$ coil, the Maxwell pair, the sample, and the radiofrequency $(\mathrm{RF})$ coil consisting of a simple two-turn transmit and receive coil tuned to $127.7 \mathrm{MHz}$, were placed in the center of the magnet bore. Before imaging, the main magnetic field was shimmed to reduce any $B_{0}$ inhomogeneity effects due to susceptibility differences caused by the gradient system and the imaging sample. The magnet bore was lined with a layer of sculpted foam in order to achieve a degree of acoustic isolation. A similar lining covered the end caps at each end of the magnet enclosing the magnet bore volume. At one end of the acoustic chamber was a linear array of electret microphones (RS Components Ltd., Corby, UK, stock no. 283-4748, with an input port diameter of $8 \mathrm{~mm}$ and an approximately omnidirectional cardioid response over $\pi$ radians), which was placed either within the acoustic chamber a distance $1.20 \mathrm{~m}$ from the gradient plate assembly and facing the front plane of the gradient coil or outside the chamber at a further distance of $10 \mathrm{~cm}$. In the latter position the linear array of microphones was used to detect the acoustic output across the diameter of the magnet approximately $1.3 \mathrm{~m}$ from the surface of the gradient coil. The linear array was comprised of 11 microphones, equally spaced $5 \mathrm{~cm}$ apart. Each microphone output was fed separately to a circuit switching arrangement, allowing manual interrogation of each microphone signal via a Hewlett Packard network analyzer. The numerical results were obtained by manually stepping through the microphone outputs, recording both the signal amplitude and phase. This procedure was used when operating in a spot frequency mode. In other experiments only the central microphone was used and the acoustic frequency stepped through a predetermined range of frequencies. The geometry of the coil design was such that a high degree of acoustic cancellation occurs along the central axis of the magnet and the acoustic noise here is consequently auto- matically low, as shown in Fig. 9. In addition, the length of the coil compared to the imaging region is such that the variation in the acoustic output vertically is expected to be small in comparison with the transverse variation. Consequently, the microphones were positioned along this transverse axis. The acoustic signal amplitudes, measured using the microphone array and network analyzer correspond to A-weighted sound pressure level (SPL) measurements in $\mathrm{dB}_{\mathrm{A}}$. (For details of our calibration method see Ref. [10].)

\section{Results}

Slice selection was set to give a coronal slice through the phantom approximately $9 \mathrm{~mm}$ thick. From the modulus image obtained, the FOV spanned 32 pixels in the frequency-encode direction and 64 pixels in the phase-encode direction. This gave the image a true resolution of $32 \mathrm{~mm} \times 64 \mathrm{~mm}$. The image was Fourier interpolated by zero filling to double the resolution along the frequency-encode axis. The structure within the phantom can be discerned down to a resolution of $2 \mathrm{~mm}$ in both directions. The image was easily corrected for the common EPI artefact of Nyquist ghosting by manually applying a first-order phase correction in the image formed from the odd echoes.

Following imaging the gradient amplifiers were reconfigured for the acoustic control experiments. The experiment was optimized by adjusting the phase and amplitude of the current in the control winding to give a noise reduction across the whole of the $x$-axis. Acoustic noise levels from the coil system were obtained using the central microphone of the array and these are plotted as a function of the current supplied to the gradient coil. Figure 5 shows the acoustic noise output in $\mathrm{dB}_{\mathrm{A}}$ versus $\log I$, where $I$ is the current in amps for the whole coil together with specific results on the whole coil at particular currents, namely 20 and $40 \mathrm{~A}$ and on a single plate pair. A least-squares fit of all the data gives the straight line $y=[21.92 x+47.018] \mathrm{dB}_{\mathrm{A}}$ with a slope of 21.92 $\mathrm{dB}_{\mathrm{A}} / \log (I)$. This demonstrates the high degree of linearity of the acoustic response with current over three orders of magnitude. The graph shows that this relationship holds for individual plate components, individual plate pairs, and the coil system as a whole.

The current required for imaging with an image acquisition time of $10.6 \mathrm{~ms}$ was $253 \mathrm{~A}$, or $\log I=2.40$. From Fig. 5 it is noted that this current corresponds to an acoustic noise output of $95.8 \mathrm{~dB}_{\mathrm{A}}$ at the operating frequency of $3.205 \mathrm{kHz}$.

Figure 6 shows the complete three-axis gradient coil assembly with a central access of $10 \mathrm{~cm}$. Figure 7 shows a detailed sketch of the full gradient assembly. Figure $8 \mathrm{a}$ is a diagram of the phantom, and Fig. $8 b$ shows the image produced. The phantom comprised a water-filled circular chamber with a diameter of $5.6 \mathrm{~cm}$, immersed within which 


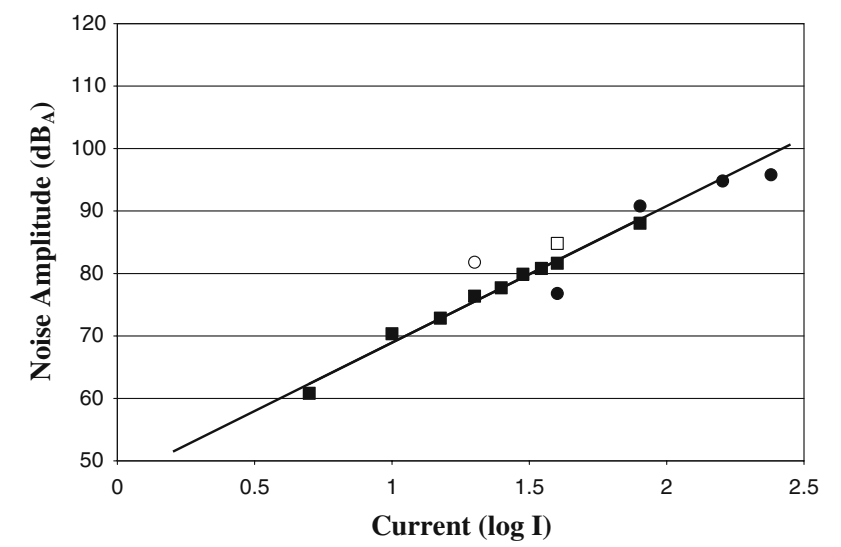

Fig. 5 Acoustic noise output in $\mathrm{dB}_{\mathrm{A}}$ versus $\log I$, where $I$ is the current in amps flowing at $3.205 \mathrm{kHz}$ for a number of coil arrangements. Closed circles show the whole coil with the full current capability. The open circle shows the whole coil with an input of $20 \mathrm{~A}$. The open square shows the whole coil response with a $40 \mathrm{~A}$ input. The closed squares show a plate pair and its response over a restricted current range

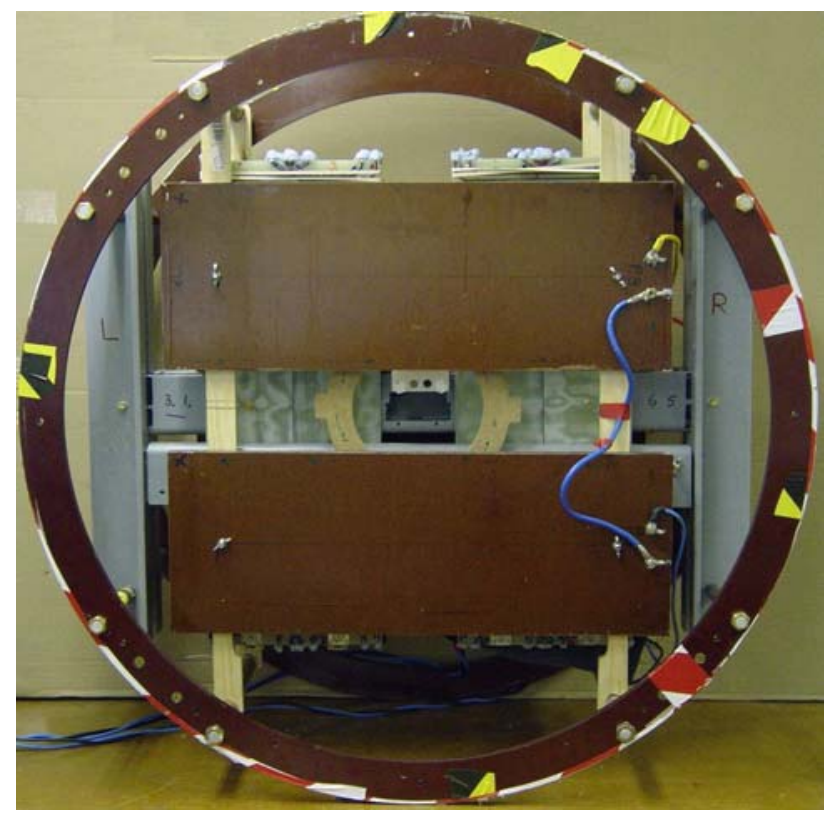

Fig. 6 Photograph of the complete coil assembly in its final form with the $G_{x}$ gradient coil plates shown at the front; the Maxwell pair and sample access can be seen in the centre

is a block of material in which various shapes and holes are machined. The water chamber is $10 \mathrm{~mm}$ thick. It can be seen in Fig. $8 \mathrm{~b}$ that there is very good agreement of the image with the small detail in the phantom. This is a one-shot image. All experiments were carried out within an acoustically lined chamber forming the inside bore of the $1 \mathrm{~m}$-bore 3.0 T magnet.

Figure 9 shows graphs of acoustic noise output versus microphone position for the complete gradient coil system shown in Fig. 6 when placed within the lined magnet bore and immersed in a $3.0 \mathrm{~T}$ static magnetic field. This noise output

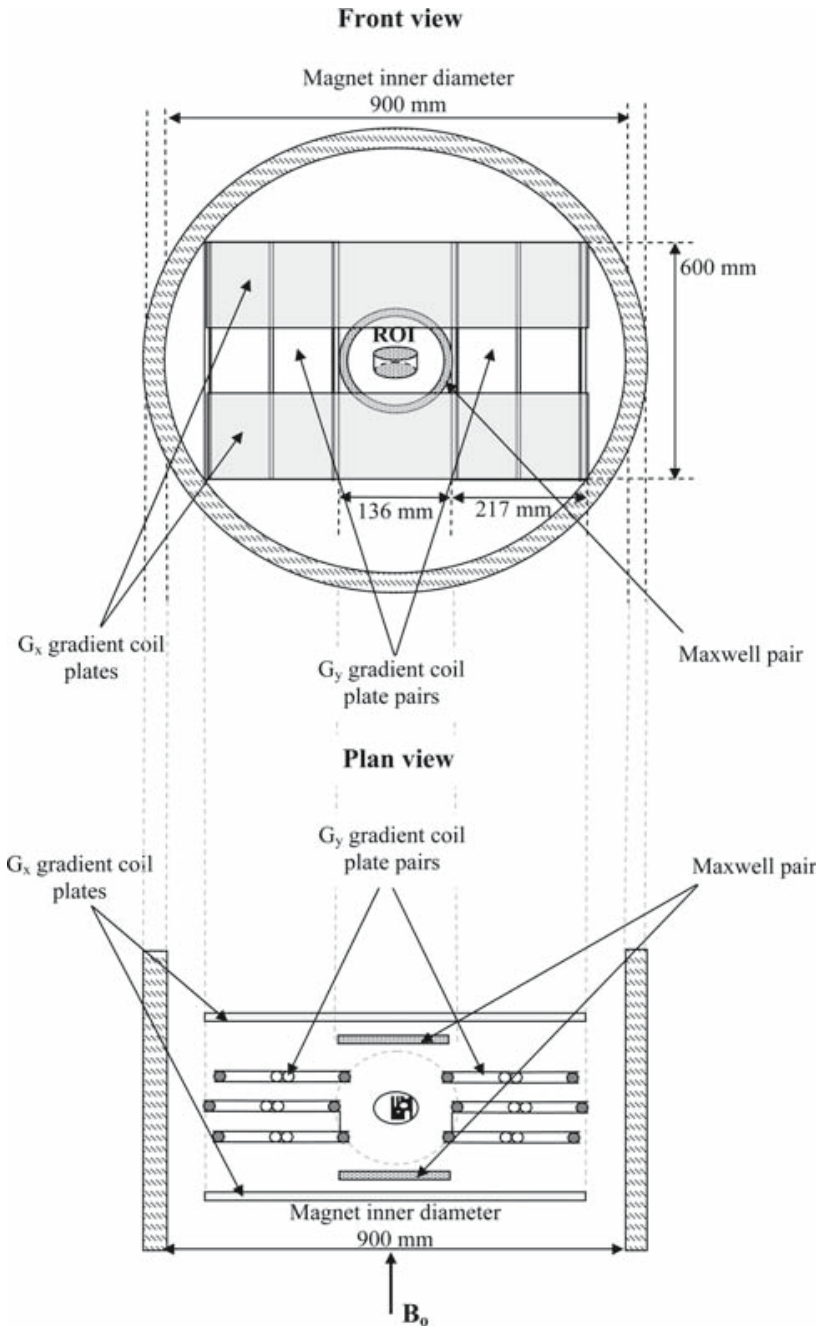

Fig. 7 Sketch of the complete coil assembly in its final form when placed in the magnet. Front view: the $G_{x}$ gradient coil plates at the front, the $G_{y}$ gradient coil plates behind, and the Maxwell pair and sample in the center. Plan view: all three gradient axes and sample in the centre

is measured using a linear array of 11 microphones placed across the magnet diameter, $1.2 \mathrm{~m}$ from the gradient coil face. Figure 9a corresponds to an acoustic operating frequency of $3.3125 \mathrm{kHz}$ with a current of $20 \mathrm{~A}$, and Fig. $9 \mathrm{~b}$ corresponds to an acoustic operating frequency of $3.3780 \mathrm{kHz}$ with a current of $40 \mathrm{~A}$. In both graphs, curves A and B correspond, respectively, to the anti-optimized high-noise output when the control current phase is $\phi=\pi$ and the optimized low-noise output when the control current phase is $\phi=0$; in both graphs the currents in the control winding and main winding were equal. The red line through curve A indicates the average acoustic noise signal. Curves $\mathrm{C}$ and $\mathrm{D}$ show the measured noise output when the microphone array was placed outside the acoustic chamber at a distance of $1.3 \mathrm{~m}$ from the gradient coil face and correspond, respectively, to the anti-optimized output when the control current phase $\phi=\pi$ and the optimized low-noise 

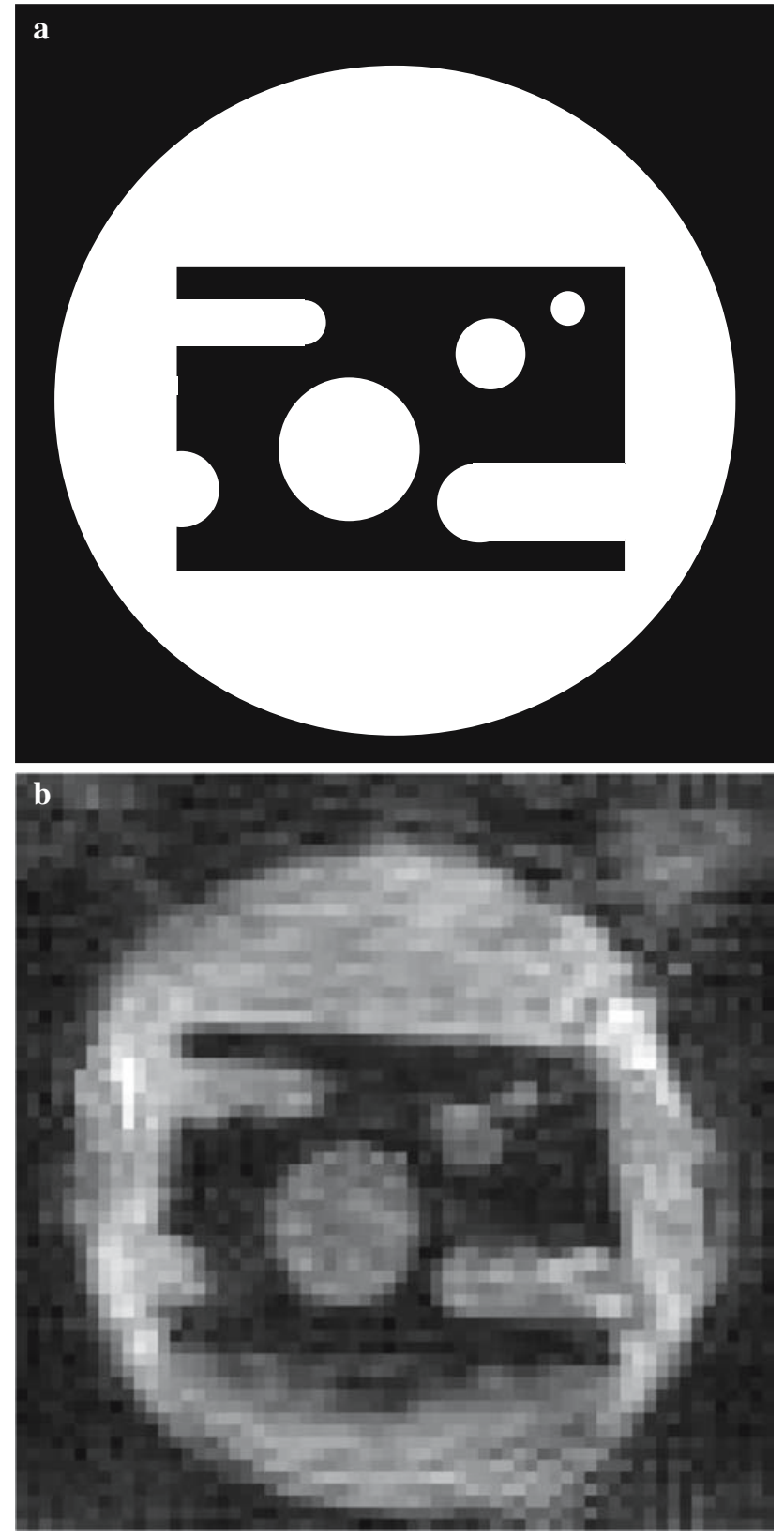

Fig. 8 a Plan view of the phantom. b Snapshot image response for the phantom, which has a machined block with cutouts and is let into the flooded water compartment. The internal diameter is $52 \mathrm{~mm}$. The circular dots have diameters of 3,6 , and $13 \mathrm{~mm}$. The image acquisition time was $10.6 \mathrm{~ms}$

output when the control current phase $\phi=0$. The red line through curve $\mathrm{D}$ shows the average acoustic noise signals. In this case approximately $20 \mathrm{~dB}_{\mathrm{A}}$ of further acoustic noise reduction is achieved and is attributable to the acoustic foam used within the acoustic chamber. The overall noise reductions achieved are substantial, amounting to $67 \mathrm{~dB}_{\mathrm{A}}$ in Fig. 9a and $60 \mathrm{~dB}_{\mathrm{A}}$ in Fig. $9 \mathrm{~b}$ relative to the control winding, which is switched in phase and in its non-optimized mode accounts for $6.0 \mathrm{~dB}_{\mathrm{A}}$ of the quoted noise reductions. a

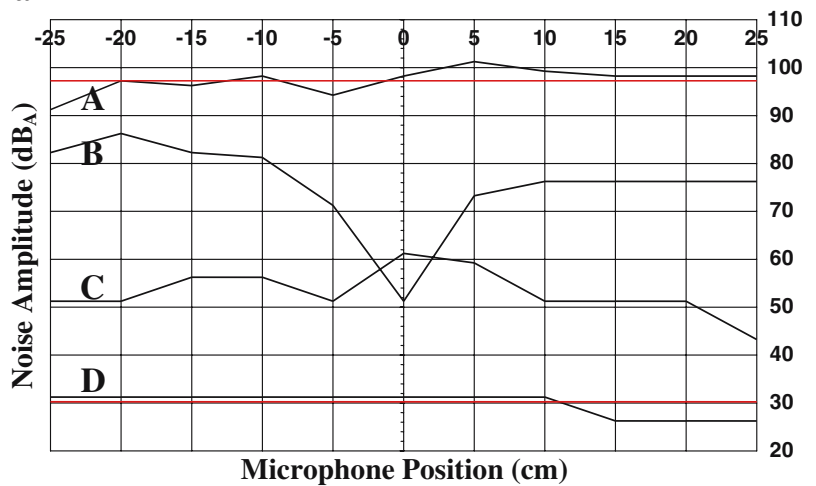

b

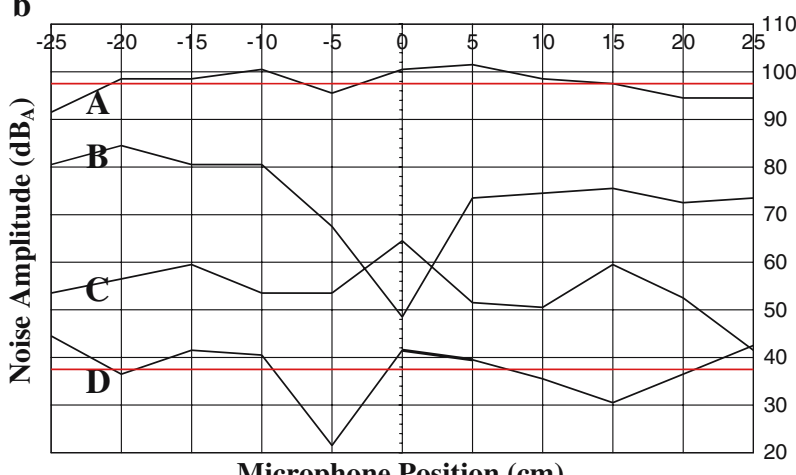

Fig. 9 Noise output data from the coil system of Fig. 6, received at the microphone array comprising 11 microphones at equally spaced positions $5 \mathrm{~cm}$ apart across the acoustic chamber ranging from $\pm 25 \mathrm{~cm}$. Curves A correspond to the antioptimised position when the control current phase $\phi=\pi$. The red line is the average of these data in both graphs. Curves $B$ show the optimised response inside the acoustic chamber and with the control current phase $\phi=0$. These curves both show an approximate $40 \mathrm{~dB}_{\mathrm{A}}$ noise reduction over the area of a centrally placed specimen. Curves $C$ correspond to the antioptimised situation when the control current phase $\phi=\pi$, with the acoustic chamber door closed and the microphone array moved just outside the acoustic chamber. Curves $D$ correspond to the optimised case when the control current phase $\phi=0$ and the microphone array is placed outside the chamber with the chamber door closed. The red line is the average of these data in both graphs. Graph a has a centre frequency of 3.3125 $\mathrm{kHz}$ and a current of $20 \mathrm{~A}$. b corresponds to $3.378 \mathrm{kHz}$ with a current of $40 \mathrm{~A}$ and is slightly above the peak in Fig. 4. The average noise reduction in $\mathbf{a}$ is $67 \mathrm{~dB}_{\mathrm{A}}$ and in $\mathbf{b}$ the average noise reduction is $60 \mathrm{~dB}_{\mathrm{A}}$.

These results are of course for noise reduction outside the magnet and indicate the effect of noise on the environment, particularly for those people standing near the magnet when in operation. However, looking in more detail at Fig. 9a, b we see that curves B in both diagrams indicate a substantial reduction in acoustic noise within the lined bore of the magnet. Typically we see a noise reduction within the acoustic chamber corresponding to $40 \mathrm{~dB}_{\mathrm{A}}$ noise attenuation over a central shaft equal to the sample diameter of $5.2 \mathrm{~cm}$. This is shown on both subfigures as the central region of curve $\mathrm{B}$. Again this figure includes the $6 \mathrm{~dB}_{\mathrm{A}}$ quoted above. 

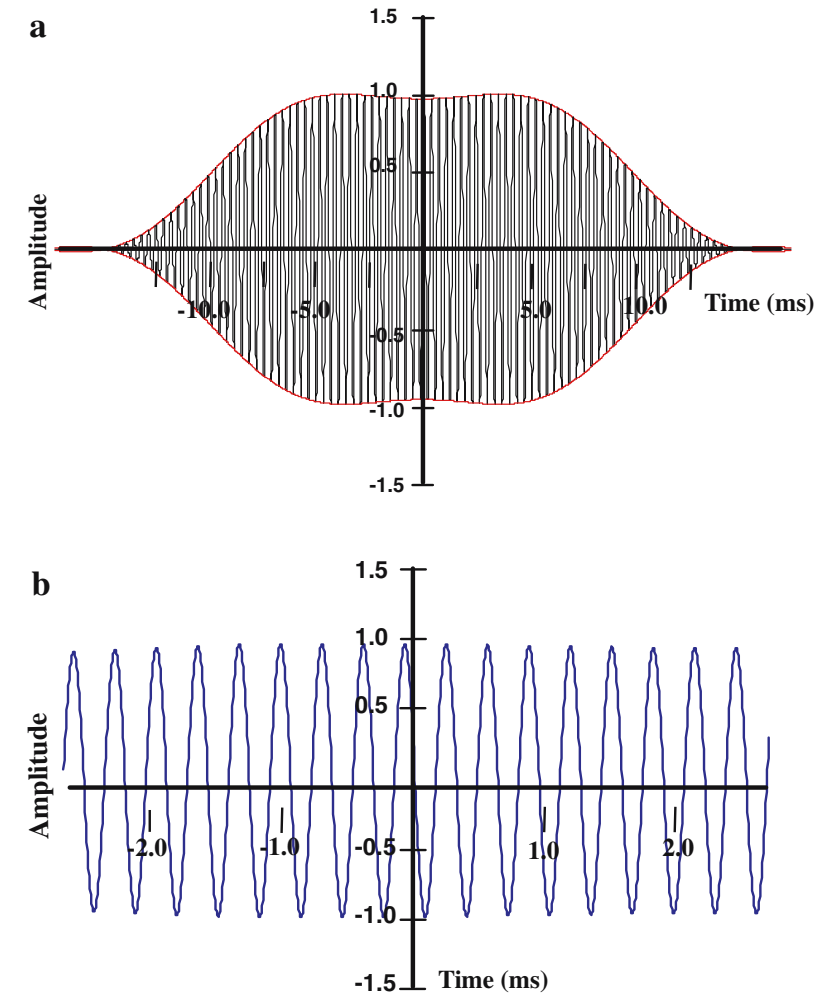

Fig. 10 a The tailored EPI pulse as used with active acoustic control, consisting of a truncated three-lobe sinc function employing five frequencies within the narrow range over which the active acoustic reduction operates. This results in a pulse that consists of a modulated sinusoidally switched gradient at the central frequency, in this case $3,205 \mathrm{~Hz}$. b One half of the central part of the symmetric tailored pulse. Allowing a two-echo redundancy before and after the necessary image data, the sampling region consists in total of 68 gradient half-cycles within the relatively flat region which is $+3.1 \%$ of the median value

\section{Discussion}

In our acoustic experiments we compared all signals with those obtained for the anti-optimum acoustic noise output level. This process seemed reasonable experimentally since it required a phase flip from the anti-optimum state to the optimum state. However this process has added an extra $6 \mathrm{~dB}_{\mathrm{A}}$ to the results, which has arguably made our results appear better.

Active acoustic control has been developed specifically to work at, or close to, the acoustic plate resonance. At frequencies far from the plate resonance we have found that the ratio of currents can deviate significantly from unity and the phase of the control current relative to the main gradient current can deviate significantly from zero. However, with the selection of suitable operating frequencies we have previously demonstrated that significant acoustic control is still possible within most regions of the acoustic spectrum [10].

As active acoustic control operates over a narrow band of frequencies a tailored switched gradient pulse was employed

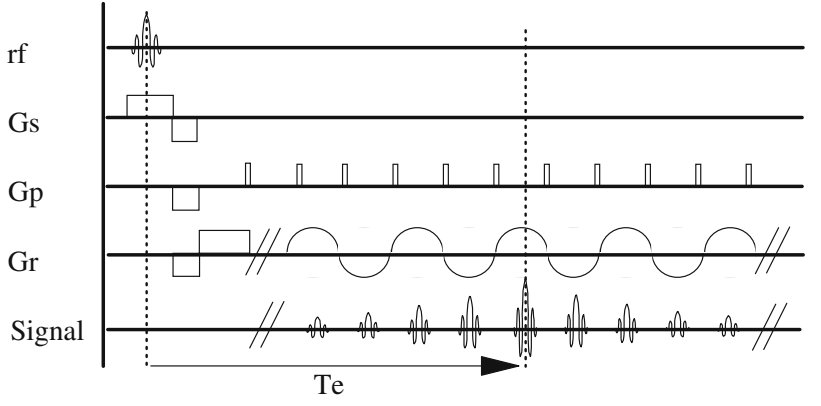

Fig. 11 Sinusoidally switched blipped EPI. This consists of a selective $90^{\circ}$ pulse applied during a coronal slice-selective gradient followed by a blipped phase encode and sinusoidally switched read gradient. Nonlinear cosinusoidal sampling was employed to match the phase evolution during the read gradient

consisting of a few frequencies within this band (Fig. 10). This produces good active acoustic noise reduction while simultaneously providing a practical sinusoidal switching gradient for high-speed EPI. Using tailored pulses of this kind has been shown to result in considerable noise reduction in individual plate pairs commensurate with that obtained in single-frequency experiments [5]. As we were not employing active acoustic control during the imaging process a simple sinusoidal switching gradient was employed in the blipped EPI sequence (Fig. 11).

The main purpose of the model coil design employed in this paper was to produce a small working coil with active acoustic control. It is impractical for use in the majority of scanners as the utilizable imaging volume is too small. While it would be possible to produce a magnetically screened version of our coil, such screening was not necessary in the present instance. Were a more practical concentric arcuate design to be implemented, including active acoustic control, then it would become essential to incorporate magnetic screening.

The greatest acoustic noise reduction occurs in the central region through the mechanism of destructive interference from each side of the coil structure, which theory tells us extends principally along the central magnet $z$-axis and gradient $x$-axis. However from our earlier work [10] it would appear that, by a suitable choice of plate material and by operating at a somewhat lower frequency commensurate with a larger object size, it will be possible to extend the gradient set, using arcuate sectors, to allow at least human head scanning and possibly whole-body imaging.

\section{Conclusion}

We have designed and built a model coil system incorporating acoustic control. This has been used to produce a snapshot image using EPI with an imaging time of $10.6 \mathrm{~ms}$. 
The gradient coil uses rectangular plate sectors that restrict the object size. The acoustic noise reduction measured over the specimen area is approximately $40 \mathrm{~dB}_{\mathrm{A}}$ as presented, or approximately $34 \mathrm{~dB}_{\mathrm{A}}$ if the acoustic control winding is not activated. Acoustic noise outside the magnet was reduced by $60-66 \mathrm{~dB}_{\mathrm{A}}$ or $54-60 \mathrm{~dB}_{\mathrm{A}}$ if the acoustic control winding is not activated.

In the future a more practical concentric arcuate design for head imaging or for whole-body access, incorporating acoustic control as well as active magnetic screening, could be built. Such a design would enable a useful comparison to be made with other whole-body gradient coil designs.

\section{References}

1. Counter SA, Olofsson A, Borg E, Bjelke B, Haggstrom A, Grahn HF (2000) Analysis of magnetic resonance imaging acoustic noise generated by a $4.7 \mathrm{~T}$ experimental system. Acta Otolaryngol 120(6):739-743
2. Price DL, De Wilde JP, Papadaki AM, Curran JS, Kitney RI (2001) Investigation of acoustic noise on 15 MRI scanners from $0.2 \mathrm{~T}$ to $3 \mathrm{~T}$. J Magn Reson Imaging 13:288-293

3. Counter SA, Olofsson A, Grahn HF (1997) Borg. MRI acoustic noise: sound pressure and frequency analysis. J Magn Reson Imaging 7:606-611

4. Hennel F, Giraud F, Loenneker T (1999) Silent MRI with soft gadient pulses. Magn Reson Med 42:6-10

5. Chapman BLW, Haywood B, Mansfield P (2003) Optimized gradient pulse for use with EPI employing active acoustic control. Magn Res Med 50:931-935

6. Edelstein WA, Kidane TK, Taracila V, Baig TN, Eagan TP, Cheng Y-CN, Brown RW, Mallick JA (2005) Active-passive gradient shielding for MRI acoustic noise reduction. Magn Res Med 53:1013-1017

7. Katsunuma A, Takamura H, Sakakura Y, Hamamura Y, Ogo Y, Tatayama R (2002) Quiet MRI with novel acoustic noise reduction. MAGMA 13:139-144

8. Mansfield P, Haywood B (2000) Principles of active acoustic control in gradient coil design. MAGMA 10:147-151

9. Pierce BO (1929) A short table of integrals. Boston, no. 162, p 24

10. Mansfield P, Haywood B, Coxon R (2001) Active acoustic control in gradient coils for MRI. Magn Res Med 46:807-818 